Z. klin. Chem. u. klin. Biochem.

8. Jg., S. 475-476, September 1970

\title{
Zusatzvorrichtung zu einem Projektions- und Ausmeßgerät für Geldiffusionsverfahren
}

\author{
Von W. Groc \\ Aus dem Heinrich-Pette-Institut für experimentelle Virologie und Immunologie an der Universität Hamburg
}

(Eingegangen am 24. April 1970)

Eine Zusatzvorrichtung zu einem Projektions- und Ausmeßgerät für Immunpräzipitate wird beschrieben. Die erreichte Verfeinerung der Meßtechnik wird am Beispiel der Erweiterung des Meßbereiches von Partigenplatten für fünf verschiedene Eiweißantigene dokumentiert.

\section{Auxillary equipment for the projection and measurement of gel diffusion patterns}

An apparatus is described for the projection and measurement of immunoprecipitates. This refinement in measuring technique is illustrated by the increased measuring range of plates for five different protein antigens.

Bei der Untersuchung eiweißarmer Körperflüssigkeiten mit Hilfe von quantitativen Geldiffusionsmethoden kann es einerseits zur Bildung sehr schwacher, andererseits zur Entstehung äußerst kleiner Immunpräzipitationsringe kommen. In beiden Fällen ist eine genaue Messung der Präzipitationsringdurchmesser (D) schwierig, manchmal sogar unmöglich.

Die üblichen Meßschablonen, Meßlupen und ähnliche Vorrichtungen, die bei routinemäßigen Gehaltsbestimmungen einer großen $\mathrm{Zahl}$ von Proteinantigenen im Blutserum ausreichend genaue Messungen gestatten, vermögen es nicht, präzis und reproduzierbar, so feine Größenunterschiede zu erfassen wie dies zum Beispiel bei Untersuchungen von nicht eingeengtem Liquor cerebrospinalis mit Hilfe verschiedener Methoden der radialen Immundiffusion $(1,2,3)$ notwendig ist. Für solche Zwẹcke verwendet man am besten Projektionsgeräte mit Mattscheibe und Meßvorrichtung.

Über das Problem der Auswertung besonders schwacher Immunpräzipitate wurde von uns an anderer Stelle berichtet (4). Zweck der vorliegenden kurzen Mitteilung ist die Beschreibung einer Zusatzvorrichtung zum Multiskop ${ }^{1}$ ), durch die der Vergrößerungsfaktor vom 12 fachen auf das 17,4 fache erhöht wird. Wir ergänzten hierzu das oben erwähnte Gerät mit Vorsatzlinsen, einem Objekthalter und einer Hebevorrichtung. Diese setzten wir aus den Teilen eines Mikroskop-Kreuztisches zusammen und befestigten sie gemeinsam mit der abnehmbaren Deckplatte an der Vorderseite des Multiskops.

Ergänzt man das Objektiv des Multiskops mit Vorsatzlinsen, ist es notwendig, die Entfernung zwischen Präparat und Objektiv zu ändern, damit das Bild auf der Mattscheibe des Projektionsgerätes scharf eingestellt werden kann. Zur reproduzierbaren Höheneinstellung dienen Skala und Nonius des verwendeten Kreuztischteiles. Den Schneckengang des Multiskopobjektivs ver-

1) Desaga, Heidelberg. sahen wir ebenfalls mit einer Millimetermarkierung. Beide Skalenteilungen dienen bei Wiederholungs- oder Reihenmessungen dazu, in Absolutwerten reproduzierbare Ergebnisse zu erhalten. Das ist besonders bei Simultanbestimmungen von Proteinantigenen wichtig, da

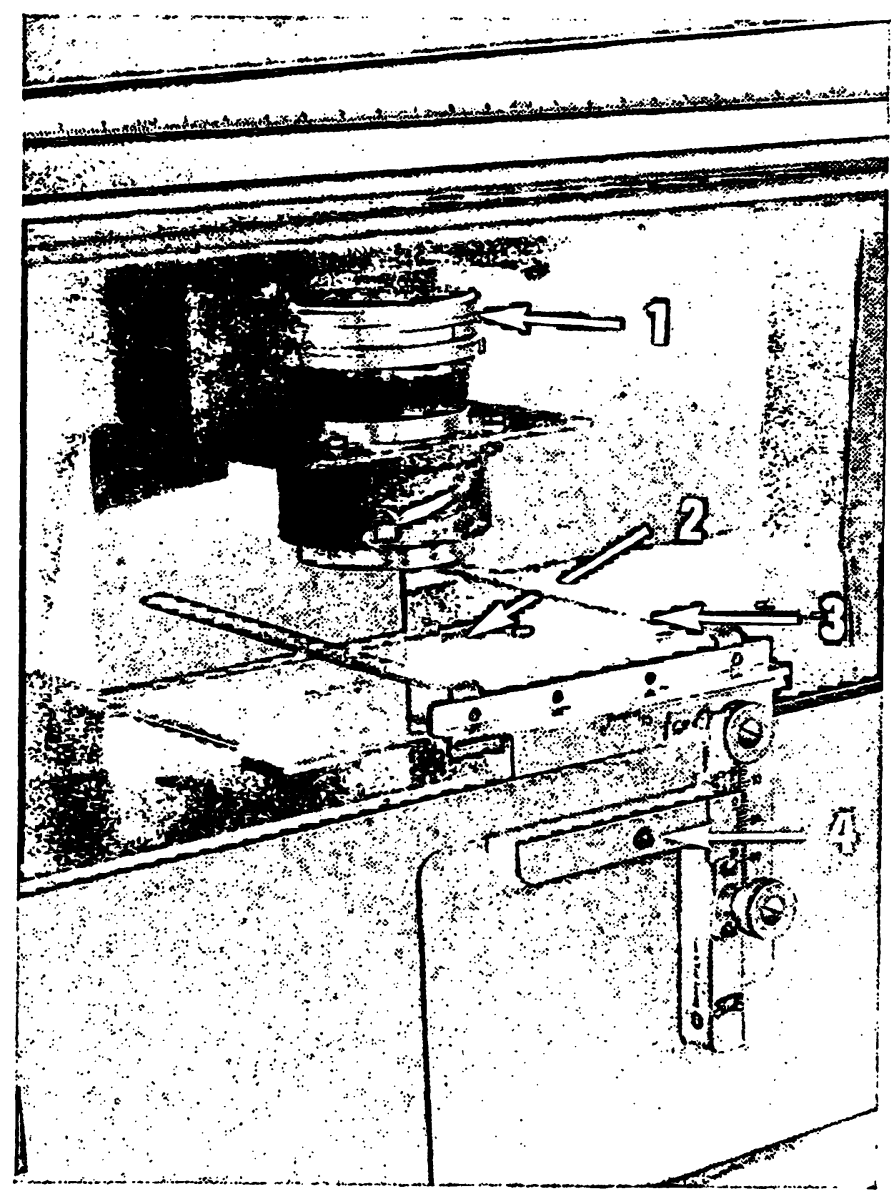

Abb. 1

Zusatzvorrichtung zum Multiskop (Desaga, Heidelberg)

1. Zusätzliche Vorsatzlinsen (Fa. B + W-Filterfabrik, Wiesbaden) 2. Glasplatte mit Fadenkreuz, dessen Schnittpunkt in der optischen 3. Abjekthalt

4. Hebevorrichtung 
hier die zur Aufstellung von Eichkurven notwendigen Proteinantigengemische verschiedener Konzentrationen sowie auch die einzelnen Analysenproben auf je einem selbständigen Objektträger oder einer separaten Plastikschale untersucht und gemessen werden $(3,5)$. Die Verwendung des bereits an anderer Stelle erwähnten Fadenkreuzes (4), dessen Schnittpunkt in der optischen Achse liegt, ermöglicht das bei unserem Arbeiten mit Vorsatzlinsen notwendige Auswerten der Präzipitatringdurchmesser in der Mitte des Blickfeldes (ohne Verzeichnung).

Nach unseren Erfahrungen (mehrere tausend Messungen) gestattet das Gerät auch mit der Zusatzvorrichtung bei Einhaltung aller optischen Kriterien ein bequemes, schnelles und zuverlässiges Arbeiten. Wir konnten zum Beispiel die untere Grenze des Meßbereiches von Partigenplatten gegenüber der vom Hersteller ${ }^{2}$ ) empfohlenen bedeutend, bei einigen Eiweißantigenen sogar um ungefähr eine Zehnerpotenz (Transferrin, Immunglobulin A) herabsetzen. Beim Präalbumin konnten wir den für unseren „Normalliquorpool“ gefundenen Wert (3) um fast $50 \%$ unterschreiten (Tab. 1).

Bei der Auswertung der Partigenplatten nach der Formel $D^{2}=k_{1} C+k_{2}$ (6) ist $z u$ beachten, daß sich die Konstante $\mathrm{k}_{2}$ bei Verwendung von Vergrößerungs-

Tab. 1

Erweiterung des Meßbereiches bei der Aufstellung von Eichkurven auf Partigenplatten

Mlit Hilfe einer Zusatzvorrichtung zum Desaga-Multiskop (Verwendet wurde stabilisiertes Standard-Humanserum Behring \begin{tabular}{c} 
Op. Nr. 369) \\
\hline Vom Hersteller Untere Meßbereich-
\end{tabular}

\begin{tabular}{lcc}
\hline & $\begin{array}{c}\text { Vom Hersteller } \\
\text { empfohlene untere } \\
\text { Meßbereichgrenze }\end{array}$ & $\begin{array}{c}\text { Untere Meßbereich- } \\
\text { grenze bei Verwen- } \\
\text { dung der Zusatz- } \\
\text { vorrichtung }\end{array}$ \\
\hline $\begin{array}{l}\text { Proteinantigen } \\
\text { Präalbumin }\end{array}$ & $\begin{array}{c}\text { in mg/100 } \mathrm{ml} \\
\text { keine Angabe }\end{array}$ & $\begin{array}{c}\text { in mg/100 } \mathrm{ml} \\
\text { bis } 0,23\end{array}$ \\
$\begin{array}{l}\alpha_{1} \text {-Antitrypsin } \\
\text { Transferrin }\end{array}$ & bis 10 & bis 2,3 \\
Immunglobulin A & bis 25 & bis 2,4 \\
Immunglobulin G & bis 15 & bis 1,8 \\
bis 15 & bis 10,0 \\
\hline
\end{tabular}

Tab. 2

Mittelwerte von $k_{2}$ für Partigenplatten in $\mathrm{mm}^{2}$ Präzipitatdurchmesser $D$ gemessen mit

\begin{tabular}{ccccc}
\hline $\begin{array}{c}\text { Meßschablone } \\
\text { Behring }\end{array}$ & Multiskop & $\begin{array}{c}\text { Multiskop } \\
\text { und } \\
\text { Vorsatzlinse } \\
\mathbf{3}\end{array}$ & $\begin{array}{c}\text { Multiskop } \\
\text { und } \\
\text { Vorsatzlinse } \\
\mathbf{4}\end{array}$ & $\begin{array}{c}\text { Multiskop } \\
\text { und } \\
\text { Vorsatzlinse } \\
\mathbf{3} \text { und 4 }\end{array}$ \\
\hline $\mathrm{k}_{2}=7,2$ & 11,02 & 15,52 & 17,05 & $\mathbf{2 1 , 7 2}$ \\
\hline
\end{tabular}

2) Behringwerke AG, Marburg/L. apparaten oder Projektionsgeräten zum Ablesen von D in Abhängigkeit vom Vergrößerungsfaktor ändert. Der Ordinatenschnittpunkt liegt bei unseren Messungen demnach höher als bei 7,2 $\pm 1,5 \mathrm{~mm}$ (6), was aus der Tabelle 2 ersichtlich ist.

Bei der graphischen Darstellung der Versuchsergebnisse nach der oben angeführten Formel ändert sich der Neigungswinkel $\alpha$ der Bezugskur,ve und damit $\mathrm{k}_{1}$, unter anderem auch in Abhängigkeit vom Maßstabverhältnis der Ordinate zur Abszisse. Bei unseren Versuchen, bei denen wir einerseits mit sehr niedrigen ProteinantigenKonzentrationen arbeiteten und andererseits die Präzipitatringdurchmesser $D$ nach entsprechender Vergrößerung bestimmten, erwies es sich als zweckmäßig, eine Maßstabänderung der Ordinaten- zur Abszissenteilung von $1: 1$ auf $1: 2,5$ vorzunehmen. Als Beispiel führen wir unsere Bezugskurve für IgA an.

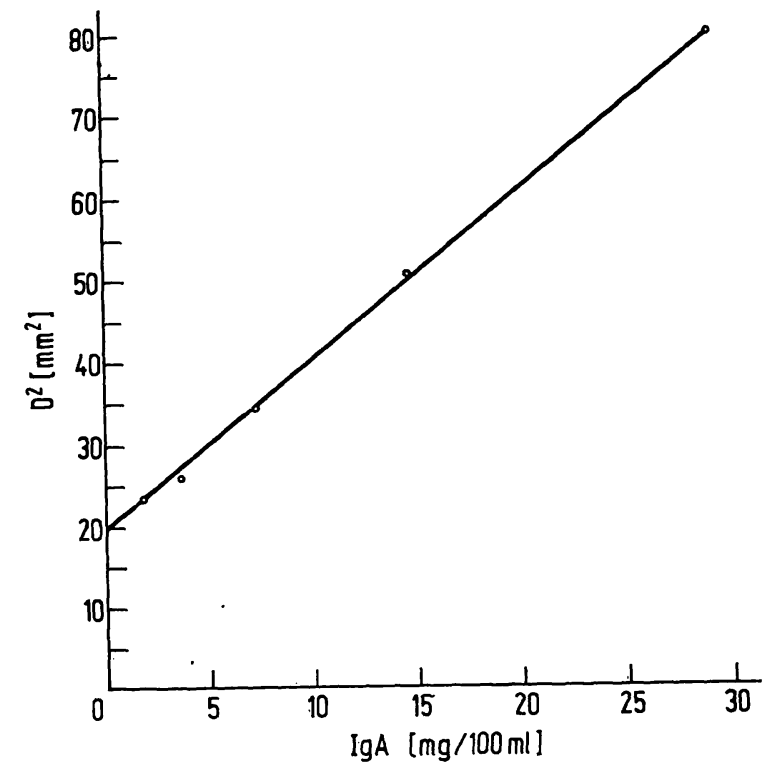

Abb. 2

Eichkurve für die Bestimmung von IgA mit Partigenplatte. Die Präzipitatdurchmesser D wurden mit dem Multiskop mit Zusatzvorrichtung ausgemessen

Abschließend bleibt noch festzustellen, daß die Ablesegenauigkeit, die auf dem Meßband des Multiskops $\sim \pm 0,25 \mathrm{~mm}$ beträgt, bei 17,4facher Vergrößerung des Präzipitatringdurchmessers noch Unterschiede von $\sim 0,029 \mathrm{~mm}$ der Originalgröße zu erfassen imstande ist.

Herrn Hermur Voss danke ich für wertvolle technische Assistenz.

\section{Literatur}

1. Laterre, E. C., H. Heulle, G. Mancini und J. F. Heremans, Protides of the biological Fluids. Section A Proteins of the Nervous System 227 (1965). - 2. FAHEY, J. L. und E. M. MC KeLVEY, J. Immunol. Baltimore 94, 84 (1964). - 3. Groc, W., Ärztl.
Lab. 16, 15 (1970). - 4. Groc, W., Das Med. Lab. (im Druck). 5. Groc, W., Kulenda, Z., Arztl. Lab. 15, 295 (1969). - 6. BECKER, W., Immunochemistry 6, 539 (1969).

Dr. W. Groc Heintich-Pette-Inst. 2 Hamburg 20 Martinistr. 52 
Differentialdiagnose von Herz- und Lebererkrankungen

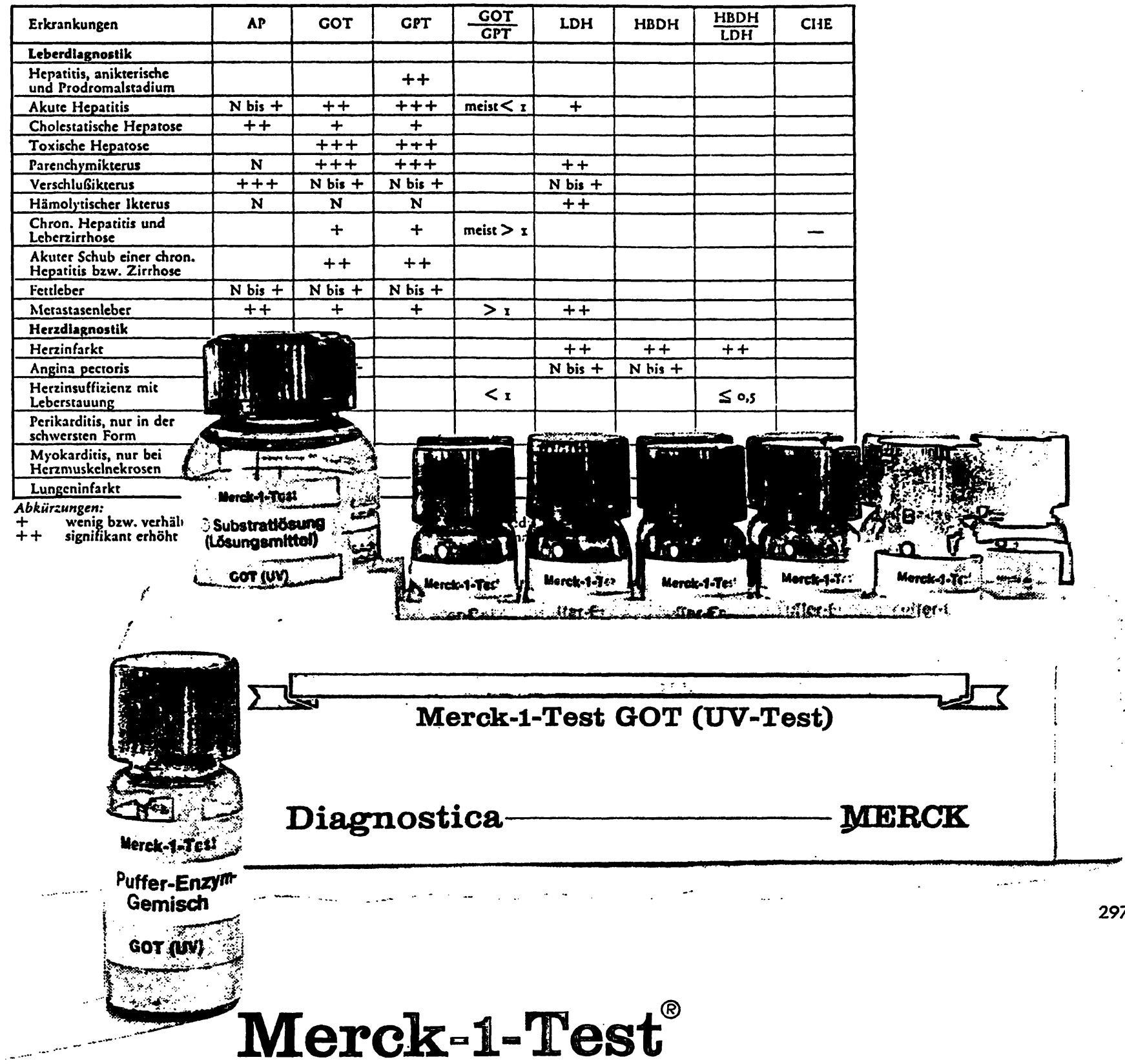

Das moderne Konzept fïr Sicherheit und Zeitersparnis in Klinik- und Praxislabor.

Merck-1-Test ${ }^{\oplus}$ Alkalische Phosphatase (kin. Test)

Merck-1-Test ${ }^{\oplus}$ Cholinesterase (kin. Test)

Merck-1-Test ${ }^{\circ}$ GOT (UV)

Merck-1-Test ${ }^{\circ}$ GPT (UV)

Merck-1-Test ${ }^{\oplus}$ HBDH (UV)

Merck-1-Test ${ }^{\oplus}$ LDH (UV)

Verlangen Sie bitte unseren Spezial-Prospekt.

E. Merck, Darmstadt 


\title{
Eine weitere Neuheit von Sigma
}

Testsätze und Reagenzien für die

\section{Qutomatisierte enzymatische Bestimmung von Glucose}

mit den Enzymen Glucoseoxydase/Peroxydase

nach dem Sigma Technical Bulletin Nr. 970

\author{
Einfach schnell
}

- empfindlich genau - zuverlässig

Keine Störung durch Harnsäure

Keine Bildung unlöslicher Farbkomplexe

Reaktionstemperatur $37^{\circ} \mathrm{C}$

Das manuelle Verfahren für die Glucosebestimmung mit Glucoseoxydase und Peroxydase hat sich als das zuverlässigste und genaueste längst durchgesetzt. Sigma ist stolz, jetzt ein enzymatisches Verfahren zur Glucosebestimmung mit dem AutoAnalyzer ${ }^{\circledR}$, Ein-, Zwei- oder SMA®-Mehrkanalgerät, bei $420-440 \mathrm{~nm}$ anbieten zu können.

Es konnte eine hervorragende Korrelation der Ergebnisse unseres neuen automatisierten Verfahrens (Bulletin 970) mit denen des manuellen enzymatischen Verfahrens (Bulletin 510) gezeigt werden.

Wir bieten an: Testsatz Nr. 970-A Anzahl der Bestimmungen: etwa 3000 Preis: $\$ 50.50$ (weniger als 2 cent pro Test)

Fracht- und portofreie Lieferung in alle Welt (wir bezahlen alle normalen Versandkosten) Sigma Technical Bulletin Nr. 970 erhalten Sie auf Anfrage

Sigma bietet bereits verschiedene manuelle Verfahren zur Glucosebestimmung an. Bitte, schlagen Sie in unserem nevesten Katalog Klinische Chemie nach oder fordern Sie Technical Bulletins Nr. 14, 510 und 635 an.

AutoAnalyzer und SMA sind registrierte Warenzeichen der Technicon Corp.

Der neve Katalog „Clinical Chemistry“ ist jetzt von Sigma erhältlich. Er enthält ein komplettes Verzeichnis aller Sigma Testsätze und Reagenzien für manuelle und automatisierte Methoden.

\section{Erbitten Sie jetzt Ihr kostenloses Exemplar.} Schreiben Sie an Department K.

Es ist ein Vergnügen mit Sigma Geschäfte zu tätigen.

Sigma-Reagenzien sind in der ganzen Welt durch den Fachhandel oder direkt aus St. Louis beziehbar.

Telegramme: SIGMACHEM, St. Louis, Missouri

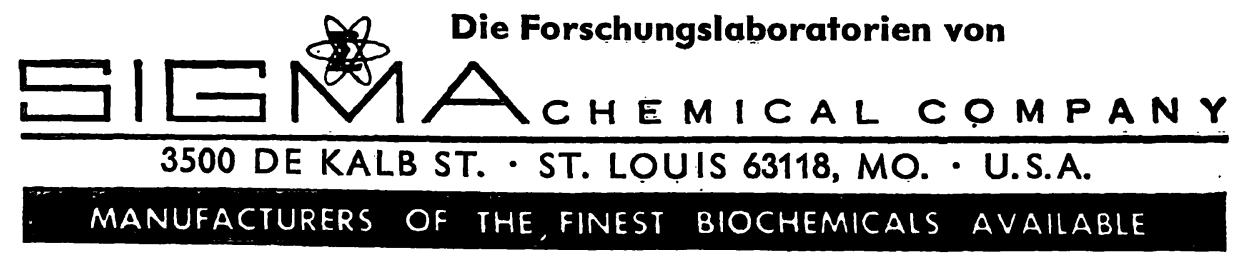

